\title{
PAN-AMPK Activation Improves Renal Function in a Rat Model of Progressive Diabetic Nephropathy $\$$
}

\author{
Xiaoyan Zhou, ${ }^{1}$ Eric S. Muise, Robin Haimbach, lyassu K. Sebhat, ${ }^{3}$ Yonghua Zhu, ${ }^{4}$ \\ Franklin Liu, Sandra C. Souza, Yanqing Kan, Shirly Pinto, ${ }^{5}$ David E. Kelley, \\ and Maarten Hoek H,6 $^{2,6}$
}

Department of Cardiometabolic Diseases (X.Z., R.H., Y.Z., F.L., S.C.S., Y.K., S.P., D.E.K., M.H.), Genetics and Pharmacogenomics (E.S.M.), and Medicinal Chemistry (I.K.S.), Merck \& Co., Inc., Kenilworth, New Jersey

Received March 20, 2019; accepted July 3, 2019

\begin{abstract}
Metabolic dysregulation and mitochondrial dysfunction are important features of acute and chronic tissue injury across species, and human genetics and preclinical data suggest that the master metabolic regulator 5 '-adenosine monophosphateactivated protein kinase (AMPK) may be an effective therapeutic target for chronic kidney disease (CKD). We have recently disclosed a pan-AMPK activator, MK-8722, that was shown to have beneficial effects in preclinical models. In this study we investigated the effects of MK-8722 in a progressive rat model of diabetic nephropathy to determine whether activation of AMPK would be of therapeutic benefit. We found that MK-8722 administration in a therapeutic paradigm is profoundly renoprotective, as demonstrated by a reduction in proteinuria $(63 \%$ decrease in MK-8722 $10 \mathrm{mg} / \mathrm{kg}$ per day compared with vehicle group) and a significant improvement in glomerular filtration rate ( 779 and $430 \mu \mathrm{l} / \mathrm{min}$ per gram kidney weight in MK-8722 $10 \mathrm{mg} / \mathrm{kg}$ per day and vehicle group, respectively), as well as improvements in kidney fibrosis. We provide evidence that the therapeutic effects of MK-8722 may be mediated by modulation of renal mitochondrial quality control as well by attenuating
\end{abstract}

fibrotic and lipotoxic mechanisms in kidney cells. MK-8722 (10 $\mathrm{mg} / \mathrm{kg}$ per day compared with vehicle group) achieved modest blood pressure reduction $(10 \mathrm{mmHg}$ lower for mean blood pressure) and significant metabolic improvements (decreased plasma glucose, triglyceride, and body weight) that could contribute to renoprotection. These data further validate the concept that targeting metabolic dysregulation in CKD could be a potential therapeutic approach.

\section{SIGNIFICANCE STATEMENT}

We demonstrate in the present study that the pharmacological activation of AMPK using a small-molecule agent provided renoprotection and improved systemic and cellular metabolism. We further indicate that modulation of renal mitochondrial quality control probably contributed to renoprotection and was distinct from the effects of enalapril. Our findings suggest that improving renal mitochondrial biogenesis and function and attenuating fibrosis and lipotoxicity by targeting key metabolic nodes could be a potential therapeutic approach in management of CKD that could complement the current standard of care.

\section{Introduction}

Individuals afflicted with chronic kidney disease (CKD) are at increased risk of cardiovascular disease and those who progress to chronic renal failure suffer increased morbidity, mortality, and a reduced quality of life. Collectively, CKD imposes a large burden on society, affecting $13 \%$ of adults in

The work was funded by Merck \& Co., Inc. Conflict of interest: All authors were employees at Merck \& Co. at the time the work was conducted and may hold stock or stock options of Merck \& Co. All authors were employees of Merck Sharp \& Dohme Corp., a subsidiary of Merck \& Co., Inc., Kenilworth, NJ at the time the work was conducted and may hold stock or stock options in Merck \& Co., Inc., Kenilworth, NJ.

${ }^{3}$ Current affiliation: Kallyope, New York, New York.

${ }^{4}$ Current affiliation: HistoBridge LLC, Hillsborough, New Jersey.

${ }^{5}$ Current affiliation: Kallyope, New York, New York.

${ }^{6}$ Current affiliation: Maze Therapeutics, Redwood City, California

https://doi.org/10.1124/jpet.119.258244.

S This article has supplemental material available at jpet.aspetjournals.org. the US, and Medicare costs for treatment of CKD were $\$ 50.4$ billion in 2011, accounting for approximately $7 \%$ of the total Medicare budget (United States Renal Data System, 2015). Current treatment options are limited to the reninangiotensin system blockers, which have modest efficacy in preventing the progression of disease. Though emerging therapies such as sodium-glucose cotransporter-2 (SGLT2) inhibitors show promise for diabetic kidney disease, other treatment approaches are urgently needed (Heerspink et al., 2018).

Multiple lines of emerging evidence have provided new insights into the role of metabolic dysregulation and mitochondrial dysfunction in the pathogenesis of CKD. Transcriptional analysis of diseased human kidneys and preclinical renal injury models showed a decrease in transcripts encoding genes involved in respiration and fatty acid oxidation (Kang et al., 2015). Likewise, peroxisome proliferator-activated

ABBREVIATIONS: AMPK, AMP-activated protein kinase; BSA, bovine serum albumin; CKD, chronic kidney disease; FDR BH, False Discovery Rate, Benjamini-Hochberg; GFR, glomerular filtration rate; GO, gene ontology; pACC, phosphorylated acetyl-CoA carboxylase; PF-06409577, 6chloro-5-[4-(1-hydroxycyclobutyl)phenyl]-1H-indole-3-carboxylic acid; PGC-1 $\alpha$, peroxisome proliferator-activated receptor gamma coactivator 1alpha; TGF, transforming growth factor. 
receptor gamma coactivator 1 -alpha (PGC-1 $\alpha$ ), a master regulator of mitochondrial biogenesis, is downregulated in diseased human kidneys as well as in animal models of acute and chronic renal injury (Dugan et al., 2013; Sharma et al., 2013). Finally, genes regulating fatty acid oxidation and mitochondrial biogenesis, such as PRKGA2, are rich in genomic loci associated with CKD (Pattaro et al., 2016). These observations prompted us to investigate the therapeutic potential of activators of 5 '-adenosine monophosphate-activated protein kinase (AMPK) in the ZSF1 rat model of diabetic nephropathy, which is a genetic model that recapitulates aspects of human metabolic syndrome with progressive kidney dysfunction. AMPK is a ubiquitously expressed heterotrimeric kinase composed of a catalytic $\alpha$-subunit and regulatory $\beta$ - and $\gamma$-subunits (Stapleton et al., 1996; Sanz, 2008; Oakhill et al., 2009; Steinberg and Kemp, 2009). AMPK functions as a master cellular energy sensor and mediates multiple effects on cellular metabolism, including regulation of glucose, lipid, and protein metabolism (Hardie et al., 2012). Importantly, AMPK activators have the potential to directly impact mitochondrial biogenesis and functionality by increasing expression of PGC- $1 \alpha$ and enhancing mitochondrial quality control by promoting mitophagy (Herzig and Shaw, 2018).

Several groups have recently reported beneficial effects of treatment with AMPK activators in models of diabetic and nondiabetic kidney disease. Nonspecific AMPK activators such as AICAR or metformin have shown renal protection in a diabetic, nonobese mouse model or nondiabetic, nonobese rat model of CKD (Dugan et al., 2013; Satriano et al., 2013). More recently, another group has reported that a $\beta 1$-subunitselective allosteric activator of AMPK (PF-06409577) was protective when administered in a prophylactic paradigm in the ZSF1 model of diabetic nephropathy (Salatto et al., 2017). We recently disclosed the first pan-AMPK activator, MK-8722 ((3R,3aR,6R,6aR)-6-((5-([1,1'-biphenyl]-4-yl)-6-chloro-1H-imidazo[4,5-b]pyridin-2-yl)oxy) hexahydrofuro[3,2-b]furan-3-ol), which has profound effects on hyperglycemia in rodent and primate models (Feng et al., 2017; Myers et al., 2017). In this report we confirm the efficacy of AMPK activators when administered therapeutically in a model with pre-existing renal disease.

\section{Materials and Methods}

Animals and Reagents. ZSF1 rats (Charles River Laboratories, Wilmington, MA), a genetic model that exhibits features of metabolic syndrome and progressive renal function decline, were used to evaluate efficacy of AMPK activator MK-8722 in our study. To minimize the potential interference of female rats' estrus cycles with study readouts, only male rats were enrolled. All rats were housed in a temperature- and humidity-controlled facility with a 12:12-hour dark-light cycle. Animals had ad libitum access to food (Purina Rodent Chow 5053; LabDiet, St. Louis, MO) and water. AMPK activator MK8722 was synthesized by Merck \& Co., Inc., Kenilworth, NJ. The structure of MK-8722 and other previously described compounds (PF06409577, AICAR and metformin) is shown in Supplemental Fig. 1. Enalapril was purchased from Sigma-Aldrich (St. Louis, MO). The medicated diets (either MK-8722 or enalapril mixed in Purina Rodent Chow 5053) were prepared by Research Diets, Inc. (New Brunswick, NJ). All procedures utilizing experimental animals were conducted in accordance with the Guide for the Care and Use of Laboratory Animals (National Research Council (US) Committee for the Update of the Guide for the Care and Use of Laboratory Animals, 2011), and experimental procedures were reviewed and approved by the Institutional Animal Care and Use Committee at Merck \& Co., Inc.

In Vivo Experimental Design. Five groups were included: lean control $(n=8)$, obese vehicle $(n=12)$, enalapril $10 \mathrm{mg} / \mathrm{kg}$ per day $(n=11)$, and MK-8722 1 and $10 \mathrm{mg} / \mathrm{kg}$ per day $(n=12$ for each dose group). Our previous experience in ZSF1 rat studies suggested that 8-12 animals per group would probably be required to assess in vivo efficacy of selected agents. We included enalapril, a standard of care agent for chronic kidney disease, as a comparator. The dose of enalapril ( $10 \mathrm{mg} / \mathrm{kg}$ per day) was selected on the basis of its beneficial renal effects in our previous studies. The doses of MK-8722 were selected based on a pilot study determining target engagement indicated by levels of phosphorylated acetyl-CoA carboxylase (pACC) in kidney, as previously described (Myers et al., 2017). The compounds were administered by in-feed dosing; compound in-feed concentration was determined by body weight and daily food intake. The treatment lasted 28 weeks starting at 20 weeks of age. We rigorously designed the study in a therapeutic mode, i.e., the treatments were initiated after the disease had developed. ZSF1 rats showed metabolic syndrome phenotype and proteinuria at 20 weeks of age, and the disease progressed over time; glomerular filtration rate (GFR) was reduced by approximately $50 \%$ at 44 weeks of age (Zhou et al., 2017). Thus, our study lasted for 28 weeks (by age of 48 weeks) to longitudinally monitor all study endpoints. All rats were acclimated to single housing in metabolism cages with free access to food and water for at least 3 days before urine and blood collection. The study rats were housed in individual home cages prior to/after metabolism collections. Twentyfour-hour urine collection was made at weeks $-2,0,2,4,6,8,12,16$, 20,24 , and 28. Urine was collected at room temperature and urine volume was recorded for each rat; blood samples were obtained by jugular vein puncture immediately after 24-hour urine collection. Urine and blood samples were then centrifuged, portioned into aliquots, and frozen at $-80^{\circ} \mathrm{C}$ until analyzed. Rats were weighed and food and water consumption were recorded at the same time on each occasion of urine and blood collection throughout the study. At the completion of the study, animals were euthanized by exsanguination and tissue harvest under deep anesthesia with isoflurane inhalation. Both kidneys were removed, and their weights were recorded. The kidney tissues were either placed in $10 \%$ neutral buffered formalin for histology assessment or snap frozen by liquid nitrogen for gene expression assessment or pACC assessment in the pilot study. Certified pathologists performed histologic assessment in a blinded manner.

In a separate study, blood pressure and heart rate response to enalapril or MK-8722 was recorded. Thirty-two-week-old, telemetered, male obese ZSF1 rats were administered vehicle, enalapril $10 \mathrm{mg} / \mathrm{kg}$ per day, MK-8722 $1 \mathrm{mg} / \mathrm{kg}$ per day, or MK-8722 $10 \mathrm{mg} / \mathrm{kg}$ per day (daily oral gavage $n=8$ for each group) for 7 days and recovered for 5 days.

Biomarkers Measurement and Plasma Compound Level Analysis. Plasma glucose and triglyceride, urine creatinine, and total protein were measured by a Roche Modular Chemistry System (Roche Diagnostics, Indianapolis, IN). Plasma creatinine was measured by mass spectrometry (Sciex, Framingham, MA). GFR was measured by FITC-sinistrin clearance in a subgroup of each main study group. For FITC-sinistrin clearance, a miniaturized device (NIC-Kidney; Mannheim Pharma \& Diagnostics, Mannheim, Germany) was used as previously described (Schock-Kusch et al., 2011; Cowley et al., 2013). In brief, the device was affixed on a depilated region of the back while the rat was under a brief $(<5$ minutes) light anesthesia with $2 \%$ isoflurane. The rat could then move freely in its home cage. After a resting baseline period of 10 minutes, a bolus of FITC-sinistrin $(15 \mathrm{mg} / 100 \mathrm{~g}$ body weight, dissolved in $0.5 \mathrm{ml}$ sterile isotonic saline) was injected through tail vein. The excretion kinetics of FITC-sinistrin were determined using a sampling rate of 60 measurements/min with an excitation time of 10 milliseconds/ measurement. Excretion kinetics were determined for 2 hours. Plasma concentrations of tested compounds were determined by 
liquid chromatography-tandem mass spectrometry (LC-MS/MS) using an API 5000 LC-MS/MS mass spectrometer (Applied Biosystems/MDS Sciex, Foster City, CA).

Kidney Gene Expression. Gene expression analysis was performed as previously described (Schlessinger et al., 2015). Briefly, sequencing was performed using the Truseq stranded total RNA RiboZero library preparation kit (cat. no. RS-122-2201) according to the manufacturer's instructions (Illumina, San Diego, CA). The resulting cDNA libraries were sequenced on an Illumina (HiSeqTM 4000) using a 50 base paired-end run. Alignment and differential gene expression analysis was performed in Omicsoft Array Studio version 10.0.0.88. Briefly, cleaned reads were aligned to the Rat.B6.0 genome reference using the Omicsoft Aligner with a maximum of two allowed mismatches. Gene level counts, and FPKM, were determined by the OSA algorithm as implemented in Omicsoft Array Studio and using Ensembl.R82 gene models. Approximately 90\% of reads across all samples mapped to the reference genome (corresponding to approximately 40 million reads). Differential gene expression analysis was performed by the DESeq2 algorithm as implemented in Omicsoft Array Studio with the samples from the vehicle-treated obese animals serving as reference, yielding fold change and corrected $P$ values (False Discovery Rate, Benjamini-Hochberg; FDR_BH). Gene ontology (GO) term enrichment analysis was performed using the PANTHER Overrepresentation test (http://pantherdb.org, release 20181113), and the GO Biologic Process Complete dataset (release 20190101). Heatmaps and box plots were created using Omicsoft Array Studio. The raw gene expression data has been deposited into the Gene Expression Omnibus database (https://www.ncbi.nlm.nih.gov/ geo/query/acc.cgi?acc $=$ GSE134804). The samples barcode ID numbers are listed in Supplemental Table 1.

Kidney pACC Assessment. pACC was assessed by a Meso Scale Discovery (MSD, Rockville, MD) assay as previously described (Myers et al., 2017). In brief, kidney tissues were homogenized. All lysates were centrifuged at $14,000 \mathrm{rpm}$ at $4^{\circ} \mathrm{C}$ for 20 minutes, the supernatants were collected, and the total protein concentration was quantitated using the BCA protein assay (ThermoFisher, Waltham, MA). pACC was quantitated using an MSD assay (Myers et al., 2017). pACC data are presented in absolute values of electrochemiluminescence (ECL) normalized by total lysate protein.

Kidney Histology Assessment. Upon completion of the study, animals were euthanized and kidneys were collected for histology assessment. Kidney tissues were fixed in $10 \%$ formalin and then paraffin-embedded. Tissue sections were stained with hematoxylin and eosin, periodic acid-Schiff (PAS), and Masson's trichrome and evaluated under light microscope. The severity of histopathologic changes in renal tubules, interstitium, vasculature, and glomeruli were graded on a $0-5$ scale corresponding to normal, minimal, mild, moderate, marked, and severe. Sections from both kidneys were examined and the final scores composite were a composite from all sections of each slice. Collagen deposition in the kidney was graded on a $0-5$ scale corresponding to minimal, mild, moderate, marked, and severe, on the basis of the blue-stained area size and intensity.

In Vitro Cell Assays. Human Renal Proximal Tubule Epithelial cells (cat. no. CC-2553, lot no. 0000385391, and cat. no. CC-2925, lot no. 0000271627; Lonza) were passaged exactly as detailed in instructions provided by Lonza, using Renal Epithelial Cell Growth Medium (REGM) medium with single-aliquot supplements. Cells were passaged for no more than four passages. Typically, experiments were run with two donors (a 58-year-old healthy female and a 69-year-old diabetic male). Results did not differ by diabetes status and were averaged. Cells were used as provided by the supplier-no attempt was made to verify their identity as proximal tubule cells, though RNA analysis indicated that cells were E-cadherin-positive, consistent with an epithelial origin. For transforming growth factor (TGF)- $\beta$ assays, primary renal proximal tubule cells at passage four or earlier were seeded at 12,500 cells/well into tissue culture-treated 96 -well plates in $100 \mu \mathrm{l}$ REGM media containing $0.5 \%$ serum. On day two, $1 \mu \mathrm{l}$ of $100 \times$ compound dilution in dimethyl sulfoxide (DMSO) was added directly to wells for 1 hour followed by the addition of $5 \mu$ l of recombinant human TGF- $\beta 1$ (580704; Biolegend) to a final concentration of $5 \mathrm{ng} / \mathrm{ml}$. Cells were incubated for 48 hours and tissue supernatant and cell lysate were analyzed for procollagen using the Pro-collagen Type 1 (human) kit (63ADK014PEG; Cisbio) according to the manufacturer's instructions. For lipotoxicity assays, primary renal proximal tubule cells were seeded at 10,000 cells/well into white-wall tissue culture-treated 96-well plates (Costar). Sixteen hours after seeding, media was replaced with $50 \mu \mathrm{l}$ of media containing $1 \times$ compound dilutions (generated from a serially diluted DMSO stock into REGM media). After a 30- to 60-minute preincubation, $50 \mu \mathrm{l}$ of media containing $2 \times$ bovine serum albumin (BSA) or BSA-palmitate (60 or $200 \mu \mathrm{M} 2 \times$ palmitate concentration) and compound at $1 \times$ concentration were added to wells. Cells were incubated for 48 hours and caspase 3/7 activity was measured using the Caspase-Glo 3/7 assay (G8091; Promega) in triplicate for each condition.

Immunoblotting of Kidney Lysates. Kidney tissues from a subgroup $(n=5)$ of each main study group was used for immunoblotting analysis. The subgroup selection was on the basis of the kidney function data, which was equal to the average of the respective main group. The MK-8722 $1 \mathrm{mg} / \mathrm{kg}$ per day group was excluded from the immunoblotting experiment since this dose did not show kidney function improvement. Kidney tissues were pulverized in a tissue mill on dry ice. Approximately $20 \mathrm{mg}$ of powdered tissue was placed in a $1.5 \mathrm{ml}$-microfuge tube to which $200 \mu$ l of RIPA buffer (ThermoFisher) containing a $3 \times$ final concentration of the HALT protease/phosphatase inhibitors (78440; ThermoFisher) and 100 IU/ml Benzonase (E8263; Sigma). Sample were mixed by pipetting and incubated with rotation for 30 minutes at $4^{\circ} \mathrm{C}$. Samples were centrifuged at $20,000 \mathrm{~g}$ for 5 minutes at $4^{\circ} \mathrm{C}$ and the supernatant was transferred to a new tube. Fifty micrograms of total protein incubated with denaturing Laemmli sample buffer (Boston Scientific) for 5 minutes at room temperature, loaded onto Nupage Novex Bis-Tris 4\%-12\% gels and run in MOPS running buffer. Gels were transferred to nitrocellulose and probed with primary and secondary antibodies in Odyssey blocking buffer (Li-Cor) before imaging on an Odyssey gel imager. Primary antibodies used were rabbit anti- $\beta$-Actin (4970P; Cell Signaling Technologies), mouse total OXPHOS Rodent WB Antibody Cocktail (ab110413; Abcam), Rabbit anti-PINK1 (ab23707; Abcam), and mouse anti- $\beta$-Actin (3700P; Cell Signaling Technologies) all at 1:1000 dilution. Secondary antibodies were IRDye $800 \mathrm{CW}$ Goat anti-Mouse IgG and IRDye 680RD Goat anti-Rabbit IgG (Li-Cor) both at 1:20,000 dilution. Signal intensity was quantified using Image-Studio software (Li-Cor) and specific band intensity was normalized to actin signal in each lane. Actin-normalized signal for each sample across both Western blots was averaged for all five animals within a group.

Statistical Analysis. All data are presented as mean \pm S.E.M. Two-way analysis of variance (ANOVA) post-hoc Tukey or linear mixed-effect model and one-way ANOVA post-hoc Tukey were used for longitudinal and one single-time-point data comparisons, respectively. A $P$ value of $<0.05$ was considered statistically significant.

\section{Results}

Effects of MK-8722 on Renal Function and Fibrosis in ZSF1 Rat. To select doses for the chronic renal efficacy study, we assessed kidney tissue pACC as a target engagement biomarker in a subset of animals treated with MK-8722 in feed for 5 days. Our laboratory had previously demonstrated that pACC is more sensitive than pAMPK for measuring target engagement with MK-8722 (Myers et al., 2017). Consistent with previously reported data in rat skeletal muscle, levels of kidney pACC increased 2-fold in ZSF1 rats treated with MK8722 at $10 \mathrm{mg} / \mathrm{kg}$ per day (Fig. 1), indicating robust target 


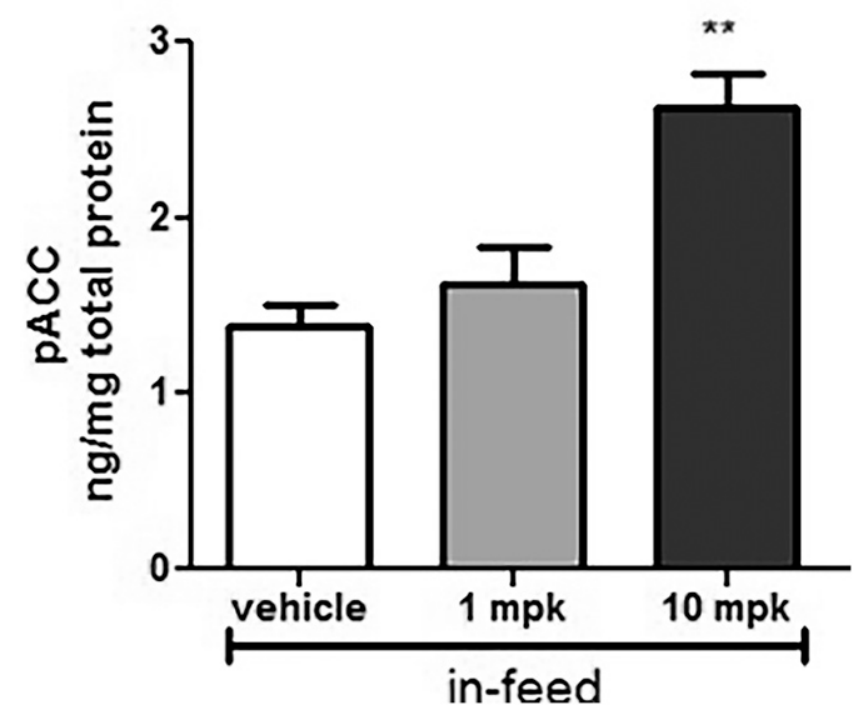

Fig. 1. Kidney target engagement after sustained AMPK activation. pACC was measured in kidney tissues harvested from animals administered MK-8722, 1 and $10 \mathrm{mg} / \mathrm{kg}$ per day in feed for 5 days $(n=5$ in each group). ${ }^{* *} P<0.01, \mathrm{MK}-872210 \mathrm{mg} / \mathrm{kg}$ per day vs. obese vehicle.

engagement in the kidney. By contrast, MK-8722 at $1 \mathrm{mg} / \mathrm{kg}$ per day had a minimal effect on pACC.

Informed by these target engagement data, we chose to test the efficacy of MK-8722, both a low and high dose, at 1 and $10 \mathrm{mg} / \mathrm{kg}$ per day in the ZSF-1 rat, a model of progressive functional renal decline associated with diabetes and metabolic syndrome. Treatment was initiated at 20 weeks of age, when renal damage was already evident as measured by proteinuria, and continued for 28 weeks. Renal function was measured by serial assessment of urinary protein creatinine ratio (UPCR) during the study as well as by measurement of
GFR using FITC-sinistrin clearance at the end of the treatment period. MK- 8722 at $10 \mathrm{mg} / \mathrm{kg}$ per day resulted in dramatic reductions in UPCR $(63 \%)$ and improvements in GFR (779 and $430 \mu \mathrm{l} / \mathrm{min}$ per gram kidney weight in MK-8722 $10 \mathrm{mg} / \mathrm{kg}$ per day and vehicle group, respectively) (Fig. 2). Moreover, MK-8722 $10 \mathrm{mg} / \mathrm{kg}$ per day significantly improved nephropathy and kidney fibrosis (Fig. 3). The effect of MK$872210 \mathrm{mg} / \mathrm{kg}$ per day on UPCR and GFR were comparable to enalapril $10 \mathrm{mg} / \mathrm{kg}$ per day.

In addition to the beneficial effects on renal function, MK8722 at $10 \mathrm{mg} / \mathrm{kg}$ per day significantly decreased plasma glucose, triglyceride levels, and body weight (Fig. 4), and lowered blood pressure (Supplemental Fig. 1). Food consumption was decreased in the first 4 weeks of treatment with MK-8722 $10 \mathrm{mg} / \mathrm{kg}$ per day but was not different from other study groups afterward (Supplemental Fig. 2). Consistent with the nominal acute target engagement data, we observed little to no effect of sustained administration of the $1 \mathrm{mg} / \mathrm{kg}$ per day dose on most metabolic parameters, nor on progression of renal disease, though a modest effect on body weight was still seen. Accordingly, hereafter, results presented will primarily focus upon those observed for MK-8722 at the $10 \mathrm{mg} / \mathrm{kg}$ per day dose.

Effect of MK-8722 in Cell-Based Models of Tubular Injury. The beneficial effects of MK- 8722 could have been attributable to normalization of systemic metabolic parameters. To investigate the potential direct renoprotective effects of MK-8722, we induced injury responses in primary proximal tubule cells via a lipotoxic or TGF- $\beta$ challenge, both of which mimic specific aspects of renal pathology relevant to diabetic kidney disease (Kang et al., 2015; Khan et al., 2018). Treatment with 30 or $100 \mu \mathrm{M}$ BSApalmitate induced substantial apoptosis as measured by the activation of caspase-3/7. These apoptotic effects were inhibited in the presence of MK-8722 (Fig. 5A). Likewise, TGF- $\beta$ induced changes in profibrotic gene expression and
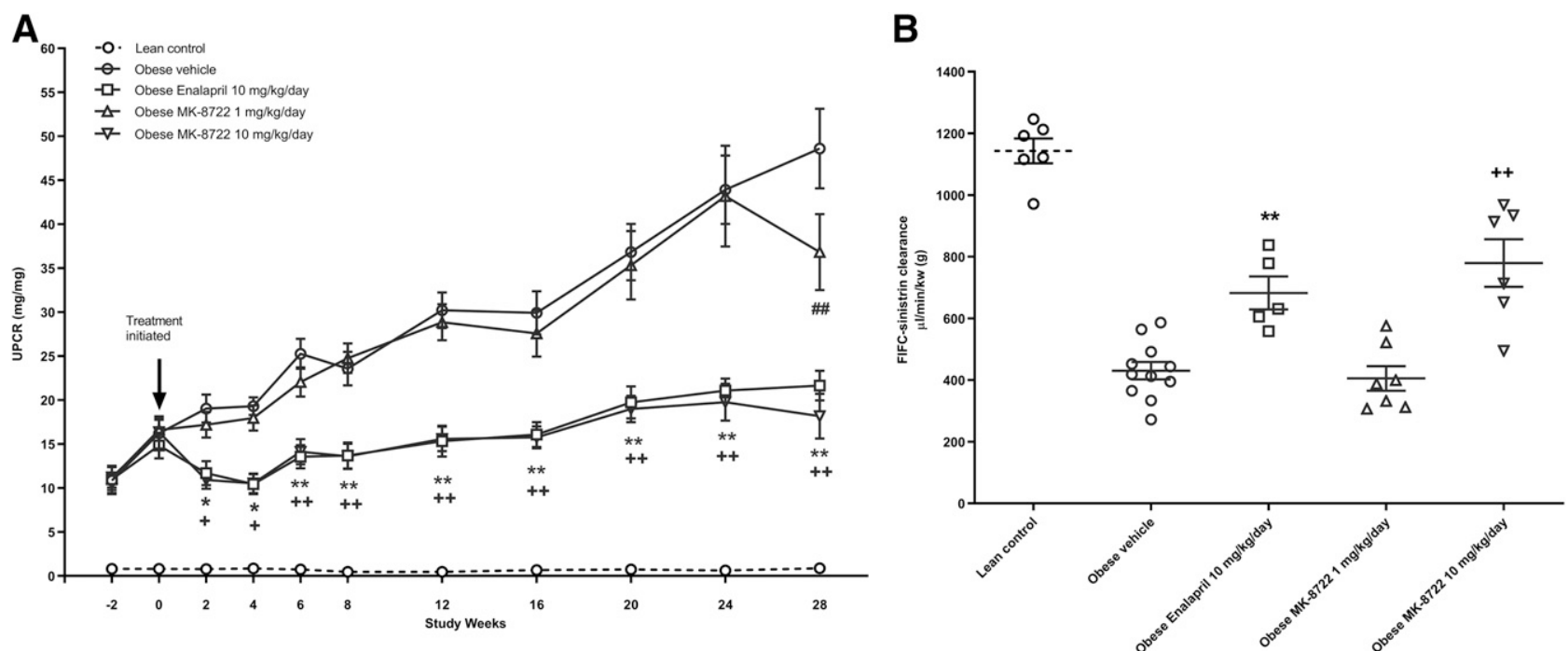

Fig. 2. (A) Time course of urinary protein-to-creatinine ratio in all groups. Data are mean \pm S.E.M. Lean control $(n=8)$, obese vehicle $(n=12)$, obese enalapril $10 \mathrm{mg} / \mathrm{kg}$ per day $(n=11)$, obese MK-8722 $1 \mathrm{mg} / \mathrm{kg}$ per day $(n=12)$, obese MK-8722 $10 \mathrm{mg} / \mathrm{kg}$ per day $(n=12) . * P<0.05 ; * * P<0.01$, enalapril $10 \mathrm{mg} / \mathrm{kg}$ per day vs. obese vehicle. ${ }^{+} P<0.05 ;{ }^{++} P<0.01$, MK-8722 $10 \mathrm{mg} / \mathrm{kg}$ per day vs. obese vehicle. ${ }^{\# \#} P<0.01$, MK-8722 $1 \mathrm{mg} / \mathrm{kg}$ per day vs. obese vehicle. (B) Glomerular filtration rate measured by FITC-sinistrin clearance. Lean control $(n=6)$, obese vehicle $(n=12)$, obese enalapril $10 \mathrm{mg} / \mathrm{kg}$ per day $(n=5)$, obese MK- $87221 \mathrm{mg} / \mathrm{kg}$ per day $(n=7)$, obese MK-8722 $10 \mathrm{mg} / \mathrm{kg}$ per day $(n=6) . * * P<0.01$, enalapril $10 \mathrm{mg} / \mathrm{kg}$ per day vs. obese vehicle. ${ }^{+} P<0.05 ;{ }^{++} P<0.01$, MK-8722 $10 \mathrm{mg} / \mathrm{kg}$ per day vs. obese vehicle. 
A
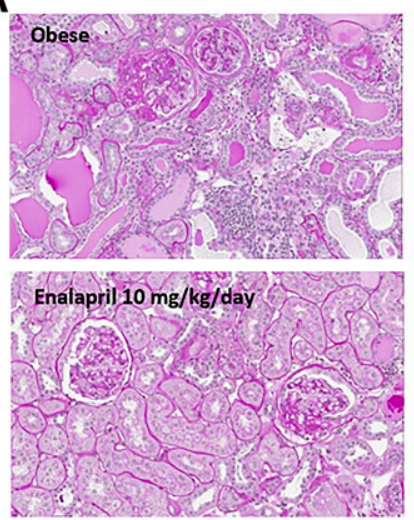

B
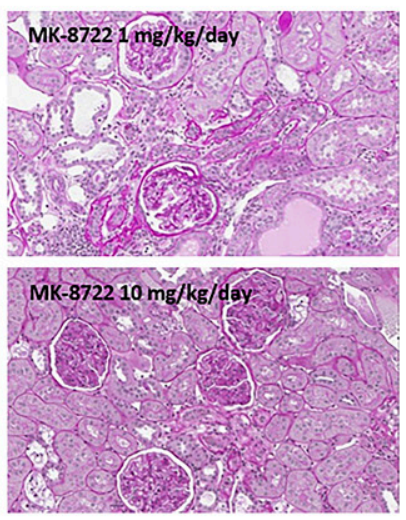
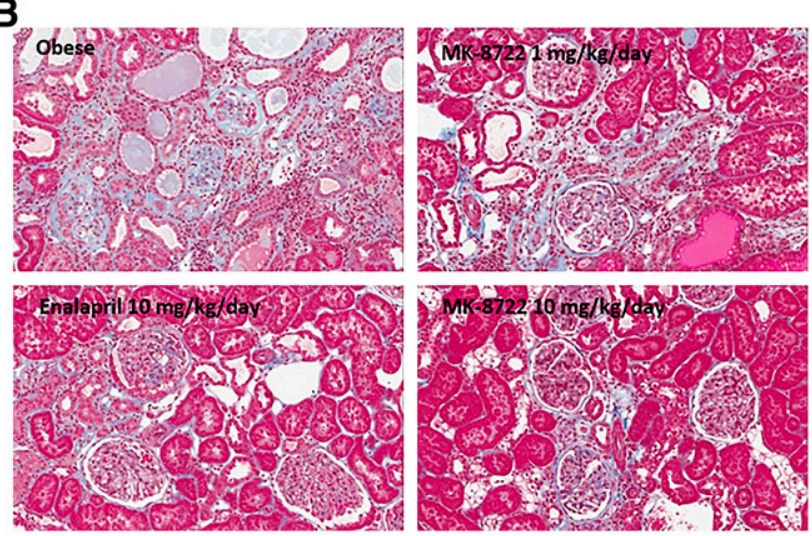

C

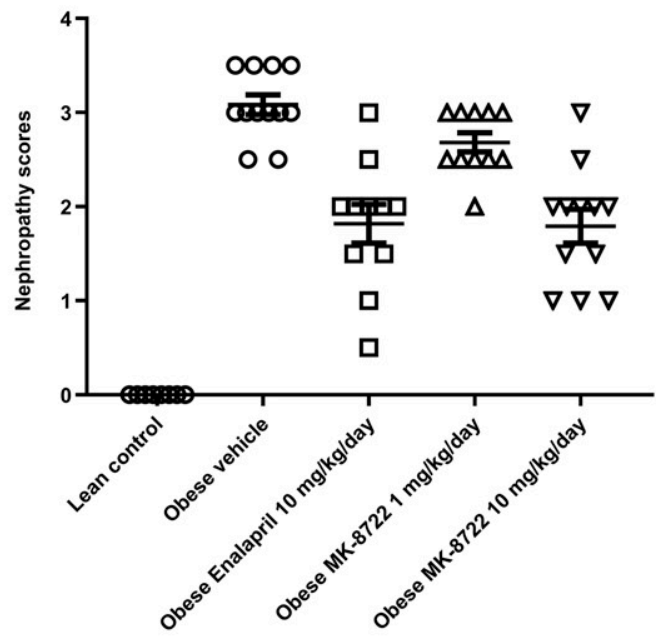

D

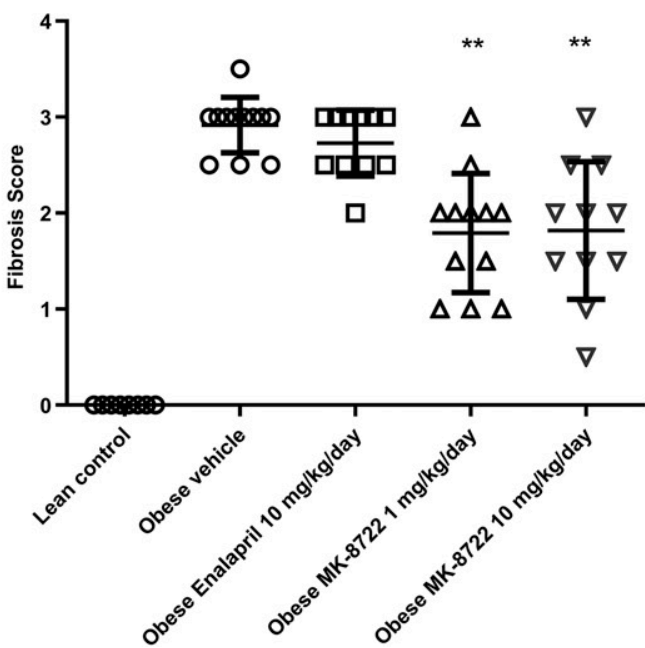

Fig. 3. (A and B) Representative hematoxylin and eosin (A) and Masson's trichrome (B) -stained sections of kidneys from obese vehicle and all three treatment groups. (C and D) Nephropathy scores (C) and fibrosis scores (D). Lean control $(n=8)$, obese vehicle $(n=12)$, obese enalapril $10 \mathrm{mg} / \mathrm{kg}$ per day $(n=11)$, obese MK-8722 $1 \mathrm{mg} / \mathrm{kg}$ per day $(n=11)$, obese MK-8722 $10 \mathrm{mg} / \mathrm{kg}$ per day $(n=12)$. ** $P<0.01$, MK-8722 $1 \mathrm{or} 10 \mathrm{mg} / \mathrm{kg}$ per day vs. obese vehicle.

procollagen production in human primary renal proximal tubule cells, which were also blocked by MK-8722 in a dosedependent manner (Fig. 5B). These results suggest that in addition to indirect effects on the kidney through improvement in metabolic parameters, MK-8722 has direct protective effects on renal epithelial cells.

Effects of MK-8722 on Kidney Gene Expression. Because of the pleiotropic effects of MK-8722 and enalapril on metabolic and/or hemodynamic parameters, we performed gene expression profiling of kidney cortex and medulla following treatment to better understand whether respective chronic effects were mediated by overlapping or orthogonal transcriptional pathways. At 48 weeks of age, there were substantial changes in obese diseased animals compared with the lean comparators, with 3150 and 4509 transcripts differentially expressed in kidney cortex and medulla, respectively (Table 1). A majority of these transcripts differentially expressed between obese diseased and lean animals were common in these two tissue sections (2559, see Supplemental Tables 3 and 6). Regarding treatment effects on transcriptional responses, treatment with MK-8722 at 10 but not $1 \mathrm{mg} / \mathrm{kg}$ per day and enalapril at $10 \mathrm{mg} / \mathrm{kg}$ per day resulted in significantly differentially expressed genes $( \pm 1.5$-fold change and FDR_BH $<0.1)$ compared with vehicle-treated obese diseased animals, and differences were observed in both cortex and medulla (532 and 344 genes in cortex and 460 and 508 genes in medulla for $10 \mathrm{mg} / \mathrm{kg}$ per day MK-8722 and $10 \mathrm{mg} / \mathrm{kg}$ per day enalapril, respectively, Table 1). Both treatments resulted in a "normalization" of the gene expression signatures, so described because of the directional change toward a "lean" transcriptional profile (Fig. 6). Comparing the transcriptional response to MK-8722 (10 mg/kg per day) with that of enalapril, we observed that only approximately $20 \%$ of the individual treatment signatures overlapped within each tissue (82 genes in cortex and 110 genes in medulla, between both compound treatments). Nonetheless, the transcriptional effects of enalapril in medulla trended toward an effect similar to that of MK-8722 treatment, especially for downregulated genes (Fig. 6, A and B). A similar trend was observed in cortex (Fig. 6, C and D). GO term-enrichment analysis of the MK8722 and enalapril signatures in medulla pointed to the nature of the differentiation in respective transcriptional responses. There was a marked effect on electron transport chain pathways by MK-8722, which was not as distinctly observed with enalapril, although more evident impacts on gene expression of diverse metabolic pathways were observed 
A

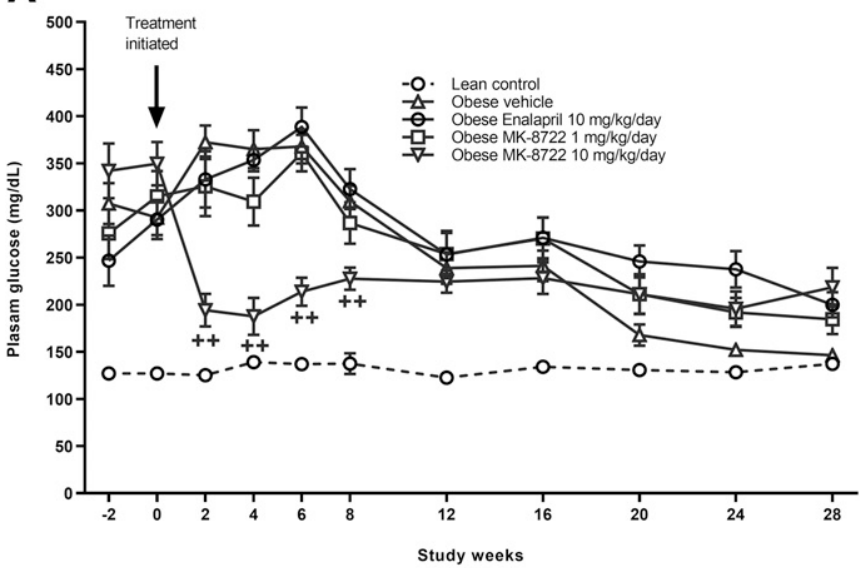

C

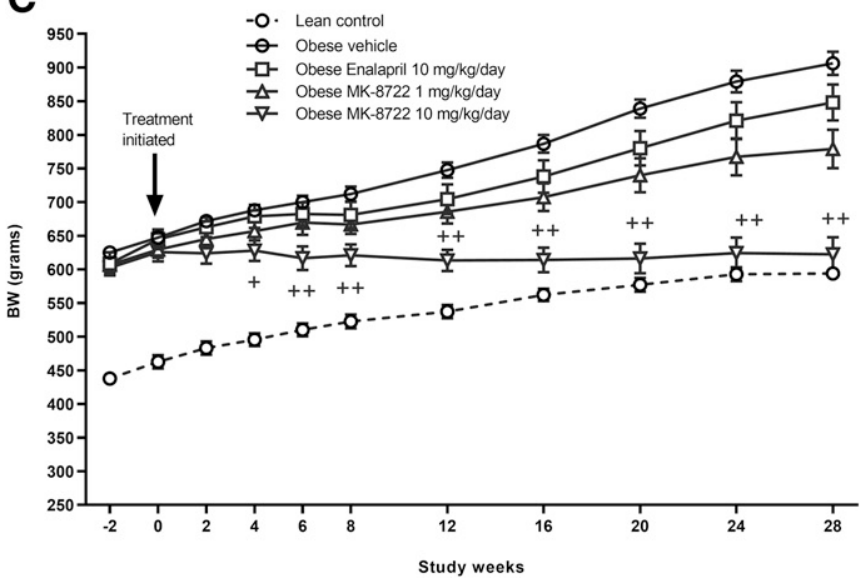

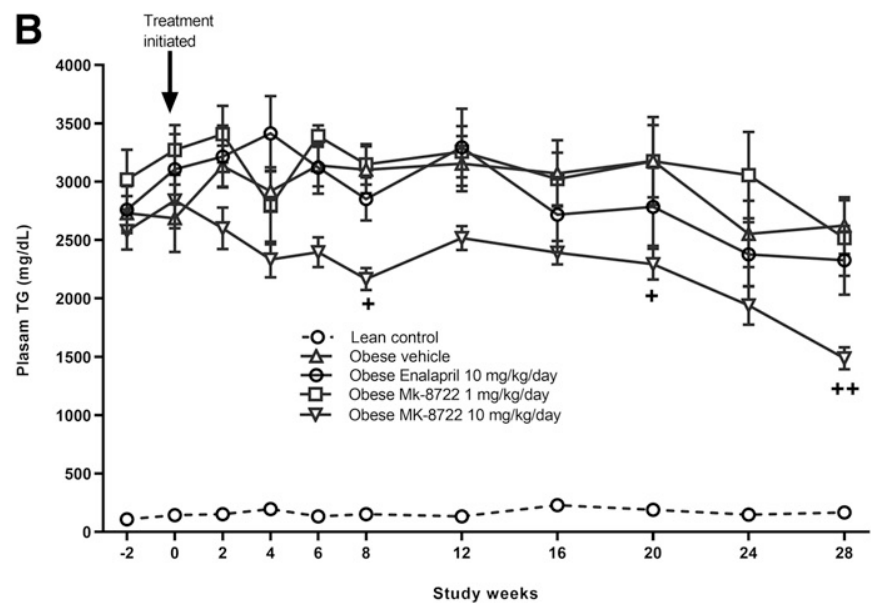

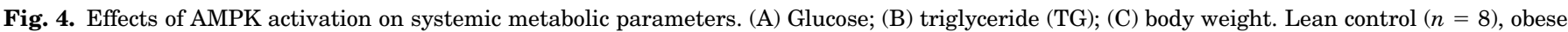

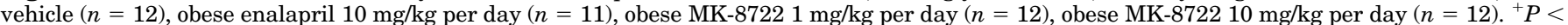
$0.05 ;{ }^{++} P<0.01$, MK-8722 $10 \mathrm{mg} / \mathrm{kg}$ per day vs. obese vehicle.

(Supplemental Tables 11 and 12; Table 2). In renal cortex, GO term-enrichment analysis also indicated some differential effects of the two treatments, with an effect on tissue development pathways by MK-8722, and upon cell-cell adhesion pathways by enalapril (Supplemental Tables 13 and 14; Table 3).

A

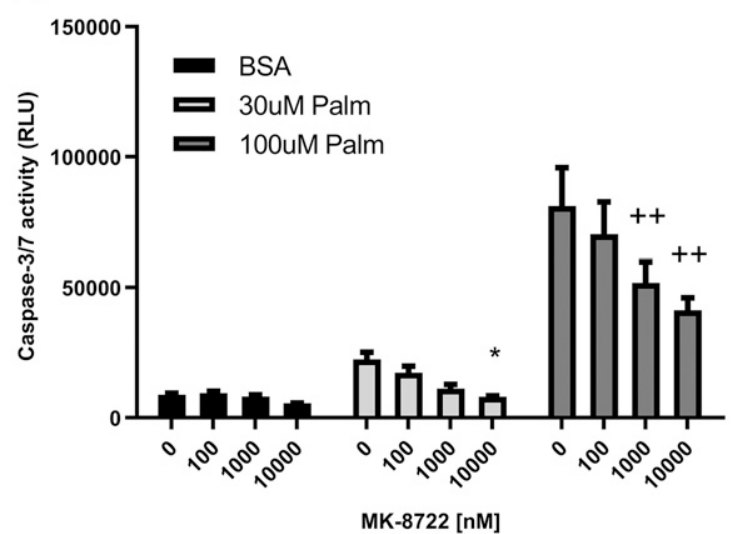

The nine genes from the respiratory electron transport chain GO term that were enriched in the MK-8722 medulla signature are shown in Fig. 7 (Supplemental Table 10), including the master regulator of mitochondrial biogenesis, PGC-1 $\alpha$, and PINK1, which plays a role in mitophagy and mitochondrial integrity (Bingol and Sheng, 2016), as well as in

B

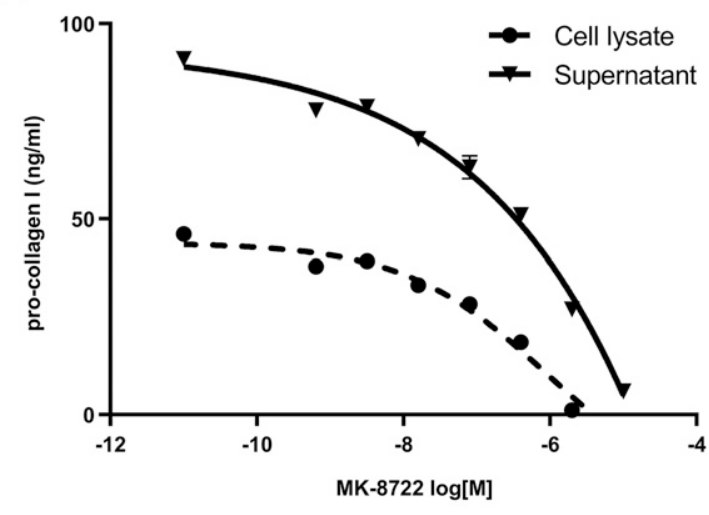

Fig. 5. AMPK activation suppresses proximal tubule injury responses in vitro. MK-8722 dose dependently blocks palmitate-induced lipotoxicity as measured by caspase-3/7 activation (A) and suppresses TGF- $\beta$ induced procollagen expression and secretion (B) in primary human renal proximal tubule cells $\left(n=5\right.$ per group). ${ }^{*} P<0.05$, MK-8722 $10,000 \mathrm{nM}$ vs. BSA (palmitate at $\left.30 \mu \mathrm{M}\right) .{ }^{++} P<0.01$, MK-8722 1000 and $10,000 \mathrm{nM}$ vs. BSA, respectively (palmitate at $100 \mu \mathrm{M}$ ). 
TABLE 1

Number of genes regulated by treatment in kidney cortex and medulla Number of genes \pm 1.5 -fold change, FDR $<0.1$ (FPKM $>1$ ).

\begin{tabular}{|c|c|c|c|c|c|}
\hline \multicolumn{3}{|c|}{ Treatment } & Baseline & Cortex & Medulla \\
\hline \multirow[t]{3}{*}{ Obese } & Enalapril & $10 \mathrm{mg} / \mathrm{kg}$ per day & Obese vehicle & 344 & 508 \\
\hline & MK-8722 & $1 \mathrm{mg} / \mathrm{kg}$ per day & Obese vehicle & 1 & 0 \\
\hline & & $10 \mathrm{mg} / \mathrm{kg}$ per day & Obese vehicle & 532 & 460 \\
\hline
\end{tabular}

anti-apoptosis and oxidative stress (Pridgeon et al., 2007). In some cases, enalapril regulated these genes in a direction similar to that of MK-8722 but not as robustly. Enalapril specifically regulated genes in some cases, such as those involved in regulation of systemic arterial blood pressure, including renin (Ren., see Supplemental Table 4). The canonical profibrotic COL1A1, COL3A1, ACTA2, TGFB2, and TGFB3, and proapoptotic transcripts CASP8 and BAD were modestly downregulated by MK-8722 $10 \mathrm{mg} / \mathrm{kg}$ per day (Supplemental Table 15).

These analyses demonstrate that despite similar efficacy, treatments with enalapril and MK-8722 were associated with normalization of mostly distinct gene expression profiles, suggesting that they act through distinct mechanisms.

MK-8722 Preferentially Normalizes Markers of Mitochondrial Dysfunction in ZSF-1 Rat Kidneys. A key potential differentiator of treatment with AMPK activators is the beneficial effects on mitochondrial function and biogenesis. Since changes consistent with enhancement in mitochondrial biogenesis were seen in the transcriptional analysis, we investigated the effects of MK-8722 treatment on electron transport chain subunits and mitochondrial quality control proteins in protein extract from kidneys. Protein components of the electron transport chain were decreased on a normalized basis in obese kidneys and restored selectively by MK-8722 but not by enalapril treatment (Fig. 8). Likewise, PINK1, a protein critical for mitochondrial quality control, was selectively restored to baseline levels by MK-8722 treatment. Together, these data suggest that MK-8722 but not enalapril treatment enhanced the functionality of mitochondria in diseased renal tissue.

\section{Discussion}

Metabolic dysregulation accompanied by defects in mitochondrial homeostasis and respiration is a key feature of acute and chronic kidney injury in humans and preclinical model systems (Kang et al., 2015; Tran et al., 2016; Khan et al., 2018). These emerging insights suggest the hypothesis that targeting key metabolic nodes that would improve mitochondrial content and function would have potential therapeutic benefit in CKD through mechanisms that are complementary but different from the effect of renin-angiotensin system blockers. This is the hypothesis tested in the current studies using the novel pan-AMPK activator MK-8722. Here we demonstrate that MK-8722, when administered in a therapeutic paradigm, can profoundly improve renal function in the ZSF-1 rat model. The effects of MK-8722 $10 \mathrm{mg} / \mathrm{kg}$ per day to improve renal function were comparable to those of enalapril $10 \mathrm{mg} / \mathrm{kg}$ per day and included an improvement in both proteinuria and GFR, though MK-8722 $10 \mathrm{mg} / \mathrm{kg}$ per day was accompanied by more significant effects on systemic metabolic parameters. Although prior reports have shown a beneficial preventative effect of direct and indirect AMPK

C

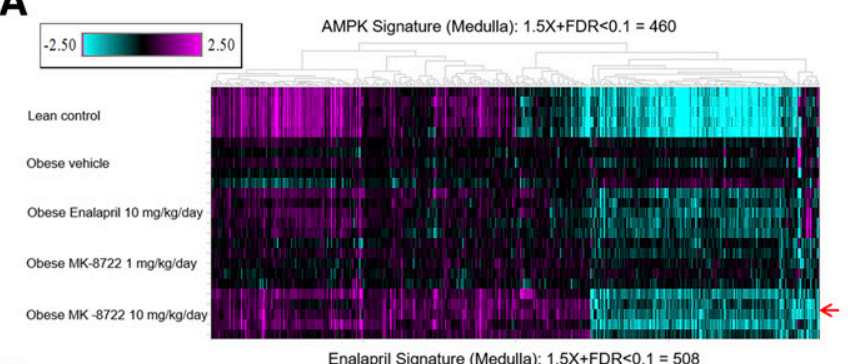

B

Enalapril Signature (Medulla): $1.5 \mathrm{X}+\mathrm{FDR}<0.1=508$

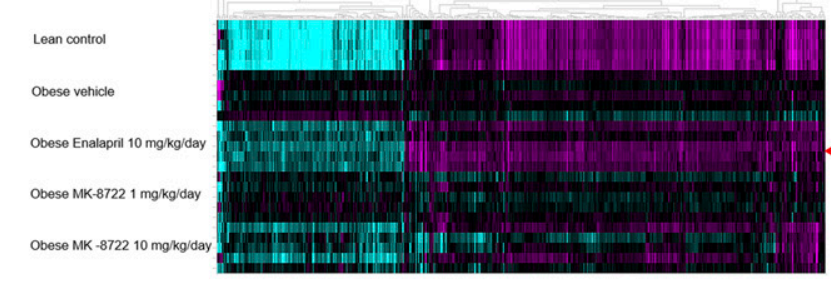

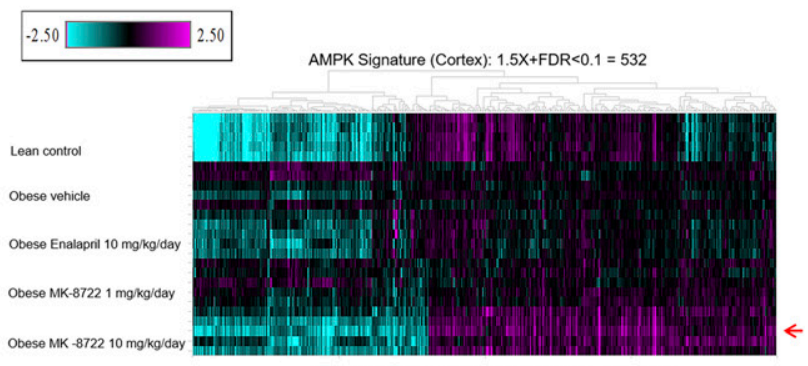

D

Fig. 6. Reversal of the disease signature in kidney cortex and medulla by enalapril or AMPK-activator treatment. Gene expression profiling of kidney medulla and cortex samples from lean control rats or ZSF1 rats treated for 28 weeks with compound ( $n=5$ per group). Shown in the heatmaps are the genes that were significantly regulated by MK-8722 $10 \mathrm{mg} / \mathrm{kg}$ per day (A and C) or enalapril $10 \mathrm{mg} / \mathrm{kg}$ per day (B and D), respectively (red arrows). The color gradient represents fold change of each individual animal (each row) compared with the pooled vehicle-treated obese animals, which served as baseline ( -2.5 to 2.5 fold). Sample details (barcodes, replicate numbers, etc., are shown in Supplemental Table 1). 
TABLE 2

GO term enrichment analysis of the medulla gene sets shown in Fig. 6A

All signature gene sets are listed in Supplemental Tables 11 and 12.

\begin{tabular}{|c|c|c|c|c|}
\hline & GO Biologic Process Complete & Fold Enrichment & Raw $P$ Value & FDR \\
\hline \multirow[t]{10}{*}{ AMPK_460 } & Electron transport coupled proton transport (GO:0015990) & 47 & $1.31 \mathrm{E}-04$ & 0.041 \\
\hline & Oxygen transport (GO:0015671) & 23 & $7.32 \mathrm{E}-05$ & 0.026 \\
\hline & ATP synthesis coupled electron transport (GO:0042773) & 7 & $1.42 \mathrm{E}-04$ & 0.044 \\
\hline & Respiratory electron transport chain (GO:0022904) & 7 & $1.67 \mathrm{E}-05$ & 0.013 \\
\hline & Proton transmembrane transport (GO:1902600) & 5 & $2.03 \mathrm{E}-05$ & 0.013 \\
\hline & Cellular respiration (GO:0045333) & 5 & $2.79 \mathrm{E}-05$ & 0.014 \\
\hline & Energy derivation by oxidation of organic compounds (GO:0015980) & 4 & $6.93 \mathrm{E}-06$ & 0.008 \\
\hline & Circadian rhythm (GO:0007623) & 4 & $5.05 \mathrm{E}-05$ & 0.020 \\
\hline & Muscle system process (GO:0003012) & 3 & $9.07 \mathrm{E}-05$ & 0.029 \\
\hline & Purine ribonucleotide metabolic process (GO:0009150) & 3 & $8.64 \mathrm{E}-06$ & 0.010 \\
\hline \multirow[t]{10}{*}{ Enalapril_508 } & Quaternary ammonium group transport (GO:0015697) & 17 & $1.87 \mathrm{E}-04$ & 0.045 \\
\hline & Hexose biosynthetic process (GO:0019319) & 12 & $2.52 \mathrm{E}-05$ & 0.010 \\
\hline & Excretion (GO:0007588) & 12 & $2.96 \mathrm{E}-05$ & 0.011 \\
\hline & Gluconeogenesis (GO:0006094) & 11 & $1.95 \mathrm{E}-04$ & 0.046 \\
\hline & Monosaccharide biosynthetic process (GO:0046364) & 10 & $6.23 \mathrm{E}-05$ & 0.020 \\
\hline & Primary alcohol metabolic process (GO:0034308) & 6 & $1.87 \mathrm{E}-04$ & 0.046 \\
\hline & Monocarboxylic acid catabolic process (GO:0072329) & 6 & $6.24 \mathrm{E}-05$ & 0.020 \\
\hline & Carbohydrate catabolic process (GO:0016052) & 6 & $2.52 \mathrm{E}-05$ & 0.011 \\
\hline & $\alpha$-Amino acid catabolic process (GO:1901606) & 5 & $1.80 \mathrm{E}-04$ & 0.046 \\
\hline & Cellular amino acid catabolic process (GO:0009063) & 5 & $8.36 \mathrm{E}-05$ & 0.025 \\
\hline
\end{tabular}

activators, the current study is the first demonstration that a direct-acting AMPK activator can modulate established renal disease when used in a therapeutic setting. As such, our study design closely mimics clinical practice for the disease stage intervention and provides further rationale for investigating the targeting of this axis in kidney disease.

RNAseq analysis of kidneys treated with enalapril and MK8722 suggested that these agents have distinct effects on the kidney. Both treatments resulted in gene expression signatures that normalized a portion of genes dysregulated in diseased versus healthy kidneys, but distinct effects were seen with each compound. Of particular note were differential changes in kidney medulla, where enalapril treatment was associated with a normalization of several metabolic processes whereas MK-8722 induced a preferential normalization of oxidative phosphorylation and pathways associated with mitochondrial biogenesis. These observations were supported by a direct measurement of components of the electron transport chain that were more significantly restored by treatment with MK-8722 than by enalapril. PINK1, a key component of mitochondrial quality control machinery was also selectively restored, at the protein and RNA level, to levels normal in kidneys of rats treated with MK-8722 but not enalapril, suggesting an overall improvement in mitochondrial health. These effects are all consistent with reported effects of AMPK on mitochondrial biogenesis and autophagy. We speculate that the combination of MK-8722 and enalapril may have additive therapeutic effects given the orthogonal mechanisms of these two different classes of therapeutically agents, which will need to be investigated in future studies.

AMPK activation has multiple effects on cellular metabolism by mediating phosphorylation of downstream proteins in catabolic or anabolic pathways (Hardie et al., 2012; (Steinberg and Kemp, 2009)), which subsequently induces glucose uptake by promoting membrane insertion of glucose transporter type 4 (GLUT4) and decreases triglyceride and cholesterol synthesis by inhibiting key lipogenic enzymes such as acetyl Co-A carboxylase (ACC), 3-hydroxy-3-methylglutary-CoA reductase

TABLE 3

GO term enrichment analysis of the cortex gene sets shown in Fig. 6B

All signature gene sets are listed in Supplemental Tables 13 and 14.

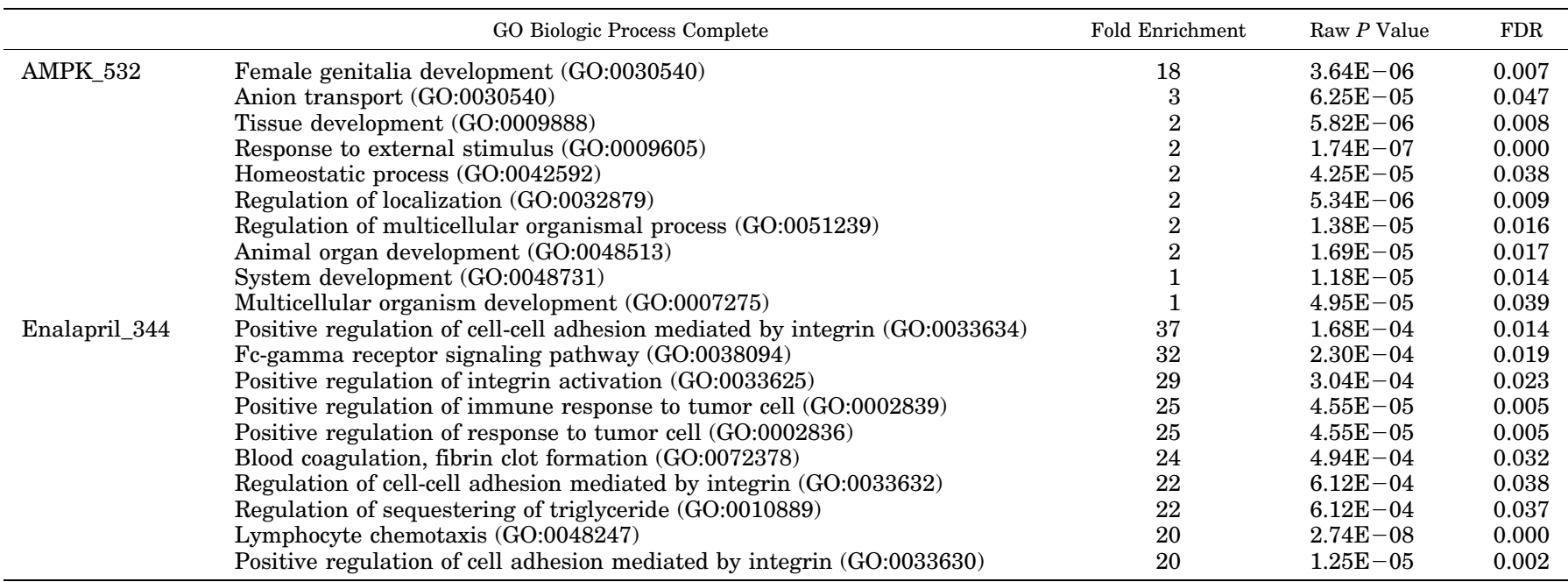




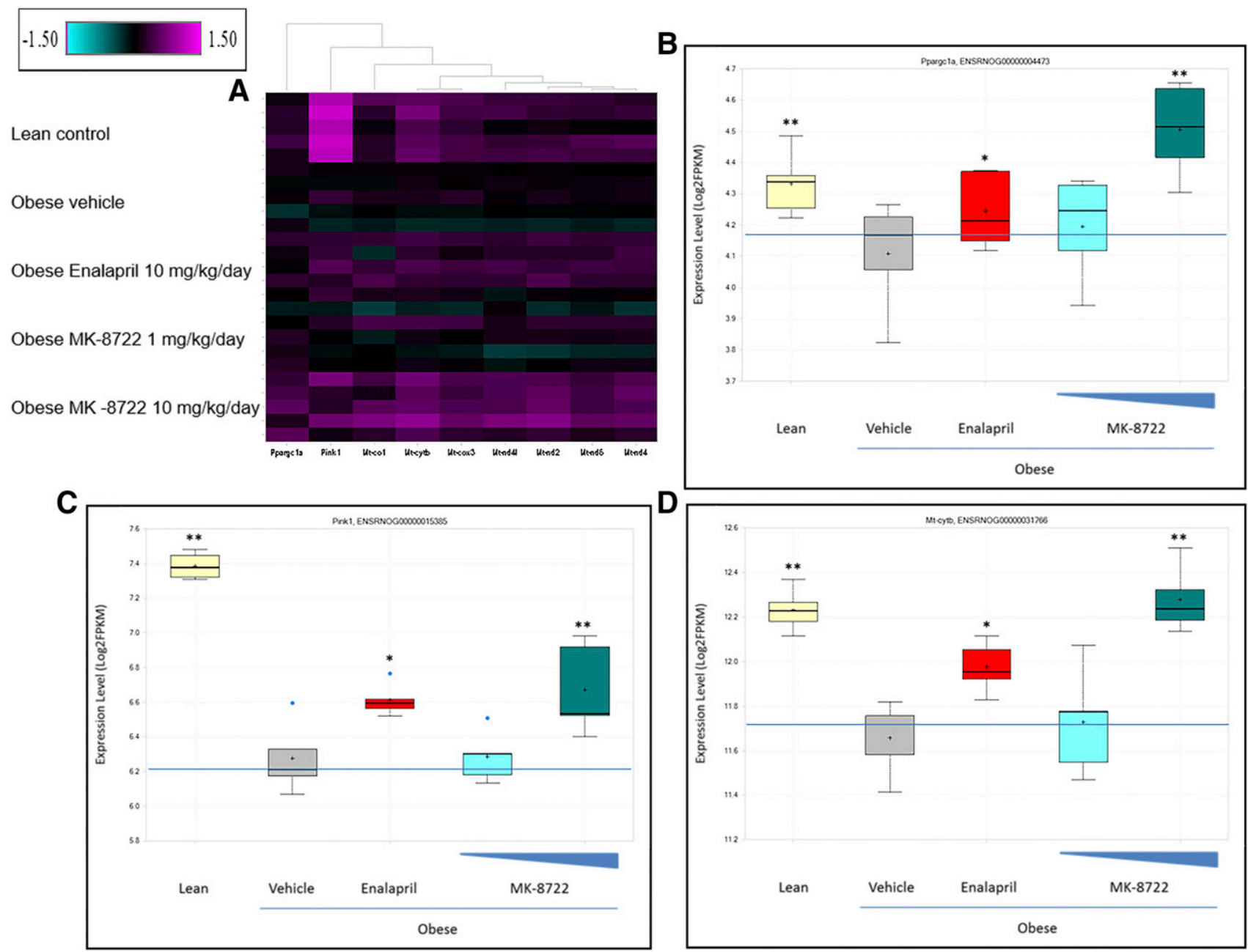

Fig. 7. Upregulation of genes involved in respiratory electron transport chain in ZSF1 kidney medulla by AMPK activation ( $n=5$ per group). Shown in the heatmap (A) are the nine genes from the respiratory electron transport chain GO term over-represented in the MK- $872210 \mathrm{mg} / \mathrm{kg}$ per day signature, as shown in Fig. 6A and Table 1. The color gradient represents fold change of each individual animal (each row) compared with the pooled vehicle-treated obese animals, which served as baseline (-1.5- to 1.5-fold). The gene list and expression data are listed in Supplemental Table 10. The box plots show the expression data for Ppargc1a (B), Pink1 (C), and Mt-cytb (D) across the treatment groups. FPKM, Fragments Per Kilobase of transcript per Million mapped reads.

(HMGR), and glycerol phosphate acyltransferase (GPAT). AMPK activation has also shown favorable effects on systemic hemodynamics by complex mechanisms, in which phosphorylation and activation of endothelial nitric oxide synthesis (eNOS) in vascular endothelium or direct relaxation of vascular smooth muscles by AMPK activation are probably involved (Buhl et al., 2002; Ford et al., 2012; Greig et al., 2015). The salutary effect of MK- 8722 in the present study on renal disease may derive partially from its impact on systemic metabolic derangements of hyperglycemia and hyperlipidemia and blood pressure. Not discounting this concept, our results also suggest that some of the beneficial effects of MK-8722 could be a result of direct action on the kidney. MK-8722 attenuated the profibrotic injury response in primary human renal proximal tubule cells and also reduced caspase activity in response to a lipotoxic challenge, suggesting that this mechanism has direct renoprotective effects on these cells. Further investigation is warranted to explain the respective contributions of AMPK-induced improvement in systemic metabolic parameters and those that derive from direct action on kidney tissue, as well as the interdigitation of both effects, to amelioration of renal disease and improvement in renal function.

Altogether, the data in the current study are consistent with a variety of independent studies demonstrating that modulation of the AMPK axis may be beneficial in kidney disease. This includes human genetics support as well as data from humans and rodent models showing that decreases in fatty acid oxidation and mitochondrial function are associated with renal dysfunction in both acute and chronic settings. Unfortunately, direct pharmacologic activation of AMPK may pose safety challenges and lack an adequate therapeutic index for chronic use in CKD. AMPK activators can cause cardiac hypertrophy in rodent models upon chronic dosing which, though reversible, does recapitulate certain features of WolffParkinson-White syndrome. This rare genetic disorder is characterized by cardiac hypertrophy, glycogen accumulation, and electrophysiological abnormalities, and can be caused by activating mutations in PRKAG2 (Porto et al., 2016). In our studies, effects of MK-8722 on heart weight/body weight ratio were noted (Supplemental Fig. 3). Interpretation of these data 

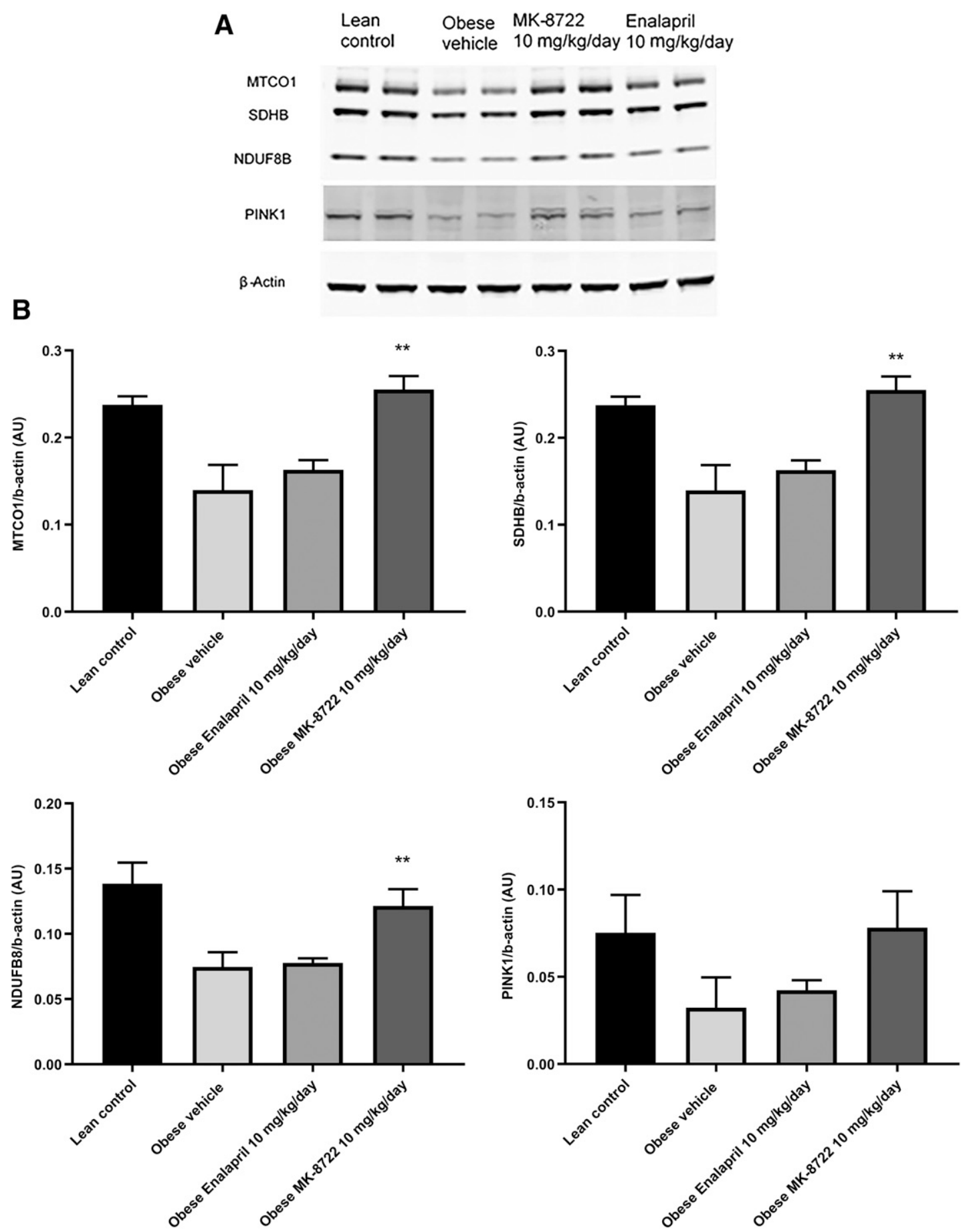

Fig. 8. Restoration of kidney mitochondrial proteins by AMPK activation. Representative immunoblots (A) and quantitation (B) of mitochondria electron transport chain (ETC) components (MTCO1, SDHB, and NDUF8B) and mitochondrial quality control proteins (PINK1) in control and treated kidneys after 28 weeks of dosing. ETC components and PINK1 are decreased in kidneys from obese animals and restored to lean control levels by AMPK activator but not by enalapril treatment. The basis of quantitation is in two independent Western blots on protein extracts from whole kidneys of five animals per group. Significance was assessed by one-way ANOVA using Dunnett's multiple comparison test using obese vehicle as control. $* * P<0.01$, MK-8722 $10 \mathrm{mg} / \mathrm{kg}$ per day vs. obese vehicle.

is complicated by the profound changes in body weight associated with treatment with MK-8722 and the lack of body weight independent normalization. However, because these effects on heart weight are consistent with our previous experience in diabetic mouse models in which there was limited weight loss, it is reasonable to infer there may be a direct effect on heart (Myers et al., 2017; Muise et al.,
2019). Interestingly, however, our laboratory recently reported that short-acting AMPK activators achieve transcriptional responses that are highly similar to those generated by longacting AMPK activators (like MK-8722) in skeletal and cardiac muscle, liver, and adipose tissue, though renal transcriptional responses were not assessed (Muise et al., 2019). Importantly, in those studies, the daily administration 
of short-acting AMPK activators did not induce cardiac hypertrophy, so this is one potential avenue for further exploration of the efficacy and safety of pharmacologic activation of AMPK in CKD.

In summary, the pan-AMPK activator MK-8722 significantly slows kidney disease progression in a rat model of diabetic nephropathy. Our findings are consistent with a large body of literature showing that modulation of metabolic axes in favor of respiration can be beneficial to the diseased kidney. Further investigation of means to restore respiratory flux in the kidney is warranted.

\section{Acknowledgments}

The authors thank E. Zycband for his technical support on processing tissue samples.

\section{Authorship Contributions}

Participated in research design: Zhou, Haimbach, Souza, Kelley, Hoek.

Conducted experiments: Zhou, Haimbach, Muise, Zhu, Liu,

Kan, Hoek.

Contributed new reagents or analytic tools: Sebhat.

Performed data analysis: Zhou, Haimbach, Muise, Zhu, Liu, Souza,

Kan, Hoek.

Wrote or contributed to the writing of the manuscript: Zhou, Muise, Haimbach, Sebhat, Pinto, Kelley, Hoek.

\section{References}

Bingol B and Sheng M (2016) Mechanisms of mitophagy: PINK1, Parkin, USP30 and beyond. Free Radic Biol Med 100:210-222.

Buhl ES, Jessen N, Pold R, Ledet T, Flyvbjerg A, Pedersen SB, Pedersen O, Schmitz $\mathrm{O}$, and Lund S (2002) Long-term AICAR administration reduces metabolic disturbances and lowers blood pressure in rats displaying features of the insulin resistance syndrome. Diabetes 51:2199-2206.

Cowley AW Jr, Ryan RP, Kurth T, Skelton MM, Schock-Kusch D, and Gretz N (2013) Progression of glomerular filtration rate reduction determined in conscious Dahl salt-sensitive hypertensive rats. Hypertension 62:85-90.

Dugan LL, You YH, Ali SS, Diamond-Stanic M, Miyamoto S, DeCleves AE, Andreyev A, Quach T, Ly S, Shekhtman G, et al. (2013) AMPK dysregulation promotes diabetes-related reduction of superoxide and mitochondrial function. $J$ Clin Invest 123:4888-4899.

Ford RJ, Teschke SR, Reid EB, Durham KK, Kroetsch JT, and Rush JW (2012) AMP-activated protein kinase activator AICAR acutely lowers blood pressure and relaxes isolated resistance arteries of hypertensive rats. $J$ Hypertens $\mathbf{3 0}$ $725-733$

Feng D, Biftu T, Romero FA, Kekec A, Dropinski J, Kassick A, Xu S, Kurtz MM, Gollapudi A, Shao Q, et al. (2017) Discovery of MK-8722: a systemic, direct pan-activator of AMP-activated protein kinase. ACS Med Chem Lett $\mathbf{9}$ 39-44.

Greig FH, Ewart MA, McNaughton E, Cooney J, Spickett CM, and Kennedy S (2015) The hypotensive effect of acute and chronic AMP-activated protein kinase activation in normal and hyperlipidemic mice. Vascul Pharmacol $\mathbf{7 4}$ 93-102.

Hardie DG, Ross FA, and Hawley SA (2012) AMPK: a nutrient and energy sensor that maintains energy homeostasis. Nat Rev Mol Cell Biol 13:251-262.

Heerspink HJL, Kosiborod M, Inzucchi SE, and Cherney DZI (2018) Renoprotective effects of sodium-glucose cotransporter-2 inhibitors. Kidney Int 94:26-39.

Herzig S and Shaw RJ (2018) AMPK: guardian of metabolism and mitochondrial homeostasis. Nat Rev Mol Cell Biol 19:121-135.

Kang HM, Ahn SH, Choi P, Ko YA, Han SH, Chinga F, Park AS, Tao J, Sharma K, Pullman J, et al. (2015) Defective fatty acid oxidation in renal tubular epithelial cells has a key role in kidney fibrosis development. Nat Med 21:37-46.
Khan S, Cabral PD, Schilling WP, Schmidt ZW, Uddin AN, Gingras A, Madhavan SM, Garvin JL, and Schelling JR (2018) Kidney proximal tubule lipoapoptosis is regulated by fatty acid transporter-2 (FATP2). J Am Soc Nephrol 29:81-91.

Muise ES, Guan HP, Liu J, Nawrocki AR, Yang X, Wang C, Rodríguez CG, Zhou D, Gorski JN, Kurtz MM, et al. (2019) Pharmacological AMPK activation induces transcriptional responses congruent to exercise in skeletal and cardiac muscle, adipose tissues and liver. PLoS One 14:e0211568.

Myers RW, Guan HP, Ehrhart J, Petrov A, Prahalada S, Tozzo E, Yang X, Kurtz MM, Trujillo M, Gonzalez Trotter D, et al. (2017) Systemic pan-AMPK activator MK8722 improves glucose homeostasis but induces cardiac hypertrophy. Science $\mathbf{3 5 7}$ 507-511.

National Research Council (US) Committee for the Update of the Guide for the Care and Use of Laboratory Animals (2011) Guide Laboratory Animals for the Care and Use of Laboratory Animals, 8th ed, The National Academies Press, Washington, DC.

Oakhill JS, Scott JW, and Kemp BE (2009) Structure and function of AMP-activated protein kinase. Acta Physiol (Oxf) 196:3-14.

Pattaro C, Teumer A, Gorski M, Chu AY, Li M, Mijatovic V, Garnaas M, Tin A, Sorice R, Li Y, et al.; ICBP Consortium; AGEN Consortium; CARDIOGRAM; CHARGeHeart Failure Group; ECHOGen Consortium (2016) Genetic associations at 53 loci highlight cell types and biological pathways relevant for kidney function. Nat Commun 7:10023.

Porto AG, Brun F, Severini GM, Losurdo P, Fabris E, Taylor MRG, Mestroni L, and Sinagra G (2016) Clinical spectrum of PRKAG2 syndrome. Circ Arrhythm Electrophysiol 9:e003121.

Pridgeon JW, Olzmann JA, Chin LS, and Li L (2007) PINK1 protects against oxidative stress by phosphorylating mitochondrial chaperone TRAP1. PLoS Biol 5: e172.

Salatto CT, Miller RA, Cameron KO, Cokorinos E, Reyes A, Ward J, Calabrese MF, Kurumbail RG, Rajamohan F, Kalgutkar AS, et al. (2017) Selective activation of AMPK $\beta 1$-containing isoforms improves kidney function in a rat model of diabetic nephropathy. J Pharmacol Exp Ther 361:303-311.

Sanz P (2008) AMP-activated protein kinase: structure and regulation. Curr Protein Pept Sci 9:478-492.

Satriano J, Sharma K, Blantz RC, and Deng A (2013) Induction of AMPK activity corrects early pathophysiological alterations in the subtotal nephrectomy model of chronic kidney disease. Am J Physiol Renal Physiol 305:F727-F733.

Sharma K, Karl B, Mathew AV, Gangoiti JA, Wassel CL, Saito R, Pu M, Sharma S, You YH, Wang L, et al. (2013) Metabolomics reveals signature of mitochondrial dysfunction in diabetic kidney disease. J Am Soc Nephrol 24:1901-1912.

Schlessinger K, Li W, Tan Y, Liu F, Souza SC, Tozzo E, Liu K, Thompson JR, Wang L, and Muise ES (2015) Gene expression in WAT from healthy humans and monkeys correlates with FGF21-induced browning of WAT in mice. Obesity (Silver Spring) 23:1818-1829.

Schock-Kusch D, Xie Q, Shulhevich Y, Hesser J, Stsepankou D, Sadick M, Koenig S, Hoecklin F, Pill J, and Gretz N (2011) Transcutaneous assessment of renal function in conscious rats with a device for measuring FITC-sinistrin disappearance curves. Kidney Int 79:1254-1258.

Stapleton D, Mitchelhill KI, Gao G, Widmer J, Michell BJ, Teh T, House CM, Fernandez CS, Cox T, Witters LA, et al. (1996) Mammalian AMP-activated protein kinase subfamily. J Biol Chem 271:611-614.

Steinberg GR and Kemp BE (2009) AMPK in health and disease. Physiol Rev 89: 1025-1078.

Tran MT, Zsengeller ZK, Berg AH, Khankin EV, Bhasin MK, Kim W, Clish CB, Stillman IE, Karumanchi SA, Rhee EP, et al. (2016) PGC1 $\alpha$ drives NAD biosynthesis linking oxidative metabolism to renal protection. Nature 531:528-532.

United States Renal Data System (2015) USRDS Annual Data Report: Epidemiology of Kidney Disease in the United States, National Institutes of Health, National Institute of Diabetes and Digestive and Kidney Diseases, Bethesda, MD.

Zhou X, Zhang J, Haimbach R, Zhu W, Mayer-Ezell R, Garcia-Calvo M, Smith E, Price O, Kan Y, Zycband E, Zhu Y, Hoek M, Cox JM, Ma L, Kelley DE, and Pinto S (2017) An integrin antagonist (MK-0429) decreases proteinuria and renal fibrosis in the ZSF1 rat diabetic nephropathy model. Pharmacol Res Perspect 5(5) DOI: $10.1002 / \operatorname{prp} 2.354$.

Address correspondence to: Dr. Xiaoyan Zhou, Cardiometabolic Diseases Department, Merck \& Co., Inc., 2000 Galloping Hill Road, Kenilworth, NJ 07033. E-mail: xiaoyan_zhou@merck.com; or Dr. Maarten Hoek, Maze Therapeutics, 123 Saginaw Dr., Redwood City, CA, 94063. E-mail: mhoek@ mazetx.com 